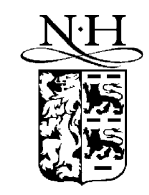

ELSEVIER

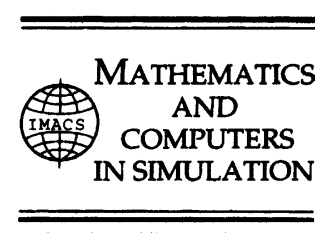

www.elsevier.nl/locate/matcom

\title{
Localized multi-dimensional optical pulses in non-resonant quadratic materials
}

\author{
Mark J. Ablowitz ${ }^{\mathrm{a}}$, Gino Biondini ${ }^{\mathrm{b}, *}$, Steve Blair $^{\mathrm{c}}$ \\ ${ }^{a}$ Department of Applied Mathematics, University of Colorado, Campus Box 526, Boulder, CO 80309-0526, USA \\ ${ }^{\mathrm{b}}$ Department of Engineering Science and Applied Mathematics, Northwestern University, \\ 2145 Sheridan Road, Evanston, IL 60208-3125, USA \\ ${ }^{c}$ Electrical Engineering Department, University of Utah, 50 S Central Campus Dr., SLC, UT 84112-9206, USA
}

\begin{abstract}
The propagation of an optical pulse in a non-resonant multi-dimensional quadratic material is studied. In a number of relevant cases, the evolution of the pulse is governed by equations of non-linear Schrödinger type with coupling to mean (i.e. low frequency) fields. The presence of this coupling can have a dramatic effect on the dynamics of the optical pulse. In particular, we show that stable localized multi-dimensional pulses can arise through interaction with boundary terms associated to the mean fields. (C) 2001 IMACS. Published by Elsevier Science B.V. All rights reserved.
\end{abstract}

Keywords: Optimal pulses; Quadratic materials; NLSM equations

\section{Introduction}

The propagation of a light pulse in optical media with quadratic non-linearity (often called $\chi^{(2)}$ materials) has attracted significant interest in recent years (see, e.g. [1,2] and references therein). In such media the non-linear response is known to generate dc fields which subsequently play a key role in the evolution of the optical pulse. In a one-dimensional non-resonant quadratic material, the evolution of the slowly-varying envelope of the optical pulse is governed $[3,4]$ by the non-linear Schrödinger equation (hereafter NLS), as in the more familiar case of centrosymmetric Kerr materials. However, it is well known that $(1+1)$-dimensional structures propagating in a multi-dimensional medium may be unstable with respect to transverse modulations (see, e.g. [5,6]). As a consequence, pulse dynamics in such multi-dimensional media cannot generally be described by simple one-dimensional systems. When studying the modulation of a wave packet in a multi-dimensional dispersive medium, generalized NLS systems with coupling to a mean term (hereafter denoted as NLSM equations) are known to appear in

\footnotetext{
* Corresponding author. Tel.: +1-847-491-3149; fax: +1-847-491-2178; URL: http://www.esam.nwu.edu/biondini. E-mail address: biondini@ @orthwestern.edu (G. Biondini).
} 
various physical situations [7,8]. In special cases these systems are known to be integrable. However, even in the non-integrable case these equations exhibit interesting phenomena such as focusing singularities and a rich structure of solutions [9].

We recently discussed [10] how novel NLSM systems appear in the context of non-linear optics when studying three-dimensional materials with quadratic non-linearity. In this letter we further develop this issue. In particular, we numerically construct solutions of these optical NLSM equations and show that the dynamics of the optical pulse is strongly influenced by the presence of the associated dc fields and, for appropriate choices of the boundary terms for the dc fields, stable localized multi-dimensional optical pulses can exist.

\section{The optical NLSM equations}

We consider the propagation of a single quasi-monochromatic optical pulse in a suitable quadratic medium (e.g., a uniaxial tetragonal $4 \mathrm{~mm}$ material like $\mathrm{BaTiO}_{3}, \mathrm{SBN}$ and $\mathrm{KTN}$ ). For convenience, we choose the coordinate axes $(x, y, z)$ to be a permutation of the crystallographic set $\left(x_{*}, y_{*}, z_{*}\right)$; i.e. we take $(x, y, z) \equiv\left(z_{*},-y_{*}, x_{*}\right)$, with the electric field polarized along $x$ and propagating along $z$. The vector non-linear wave equation which governs the evolution of the pulse is

$$
\nabla^{2} \boldsymbol{E}-\nabla(\nabla \cdot \boldsymbol{E})-\frac{1}{c^{2}} \frac{\partial^{2}}{\partial t^{2}}(\boldsymbol{E}+\boldsymbol{P})=0,
$$

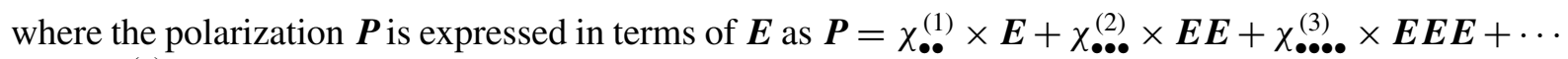
(with $\chi^{(n)}$ denoting the $n$th order susceptibility tensor of the medium) and $c$ is the speed of light in vacuum. Following standard perturbation methods associated with the derivation of NLS-type systems (see, e.g. [11]), we expand the electric field $\boldsymbol{E}$ in each Cartesian component $j$ as

$$
E_{j}=\varepsilon E_{j}^{(1)}+\varepsilon^{2} E_{j}^{(2)}+\varepsilon^{3} E_{j}^{(3)}+\cdots,
$$

where $\varepsilon$ is a small parameter that measures the non-linearity of the pulse and its narrow frequency spread around the carrier. We introduce the rapidly varying phase $\theta=k z-\omega t$ (where $\omega$ is the central frequency of the pulse and $k$ is the wavenumber), the slowly varying space coordinates $X=\varepsilon x, Y=\varepsilon y, Z=\varepsilon^{2} z$, the retarded time $T=\varepsilon\left(t-z / v_{\mathrm{g}}\right)$, and we expand the electric field $\mathrm{E}$ in harmonics as

$$
E_{j}^{(n)}(x, y, z, t)=\sum_{m=-n}^{n} \mathrm{e}^{i m \theta} E_{j, m}^{(n)}(X, Y, Z, T),
$$

where only the fundamental is present at leading order, and all the higher harmonics are generated by the non-linearity. We then substitute Eqs. (2) and (3) into Eq. (1). The main results of the analysis are summarized in the following.

At $\mathrm{O}(\varepsilon)$ we find the dispersion relation $k=\kappa_{x}(\omega)$, with the propagation wavenumber and the refraction index given by $\kappa_{j}^{2}(\omega)=(\omega / c)^{2} n_{j}^{2}(\omega)$ and $n_{j}^{2}(\omega)=1+\hat{\chi}_{j j}^{(1)}(\omega)$, respectively, where $\hat{\chi}^{(1)}(\omega)$ is the Fourier transform of $\chi^{(1)}(t)$. At $\mathrm{O}\left(\varepsilon^{2}\right)$ we find the group velocity $v_{\mathrm{g}}=v_{x}(\omega)$, with $v_{j}(\omega)=1 / \kappa_{j}^{\prime}(\omega)$, and we solve explicitly for the second harmonic in terms of the field at the fundamental, under the assumption that there are no resonant interactions. However, the expansion also allows the presence of dc fields in all Cartesian components: $E_{j, 0}^{(2)} \neq 0$. At $\mathrm{O}\left(\varepsilon^{3}\right)$, defining $A(X, Y, Z, T)=E_{x, 1}^{(1)}(X, Y, Z, T)$ and 
$\phi(X, Y, Z, T)=E_{x, 0}^{(2)}(X, Y, Z, T)$, which represent the pulse envelope and the $x$-component of the dc field, respectively, we have

$$
2 i k A_{Z}+r_{x, 1} A_{X X}+A_{Y Y}-k k^{\prime \prime} A_{T T}+M_{x, 1}|A|^{2} A+M_{x, 0} \phi A=0,
$$

with $r_{j, m}=n_{j}^{2}(m \omega) / n_{z}^{2}(m \omega)$. Finally, at $\mathrm{O}\left(\varepsilon^{4}\right)$, we find equations for all dc terms. In particular

$$
r_{x, 0} \phi_{X X}+\phi_{Y Y}+s_{x} \phi_{T T}=N_{x, 1}\left(|A|^{2}\right)_{T T}-N_{x, 2}\left(|A|^{2}\right)_{X X},
$$

with $s_{j}=1 / v_{j}^{2}(\omega)-1 / c_{j}^{2}(0)$, with the phase velocity given by $c_{j}^{2}(\omega)=c^{2} / n_{j}(\omega)$. Subscripts on $A$ and $\phi$ denote partial derivatives.

\section{Remarks}

Eqs. (4a) and (4b) constitute the fundamental system of $(3+1)$-dimensional equations that govern the evolution of the slowly varying envelope of the optical pulse. They are the optical analogue of the $(2+1)$-dimensional Benney-Roskes equations in water waves [7]. The coefficients $M_{x, m}$ and $N_{x, m}$ (cf. [10]) depend on the material considered. In particular, $M_{x, 1}$ contains the third-order self-phase modulation coefficient plus the effective contribution due to second harmonic cascading, $M_{x, 0}$ is the second-order coupling coefficient between dc and fundamental and the $N_{x, m}$ are the second-order coefficients of the dc rectification process, while the $r_{x, m}$ are affected by the contribution of the divergence of the electric field. In what follows we will consider the case $s_{x}<0$.

It is important to note that: (i) Eqs. (4a) and (4b) are derived under the assumption that there are no resonant wave interactions. As mentioned above, in this case the second harmonic can be solved explicitly in terms of the field at the fundamental [10]. Otherwise the governing equations and relevant scales are very different, e.g. two/three wave interactions, which have already been the subject of many research papers (see, e.g. [12,13] and references therein). (ii) We do not introduce a dc field and/or second harmonic at leading order because we are interested in the evolution of a modulated optical field and not in the interactions amongst different waves. This is a standard assumption in order to obtain NLS - and in this case NLSM - type equations. Of course, other assumptions would lead to different evolution equations (cf. $[6,9,14,15])$. (iii) The dc field needs to be included in the calculation, otherwise inconsistencies arise in the derivation of the evolution equations. This is true even in the one-dimensional case, where, however, the mean fields can be integrated explicitly, and the equations reduce to the NLS equation (cf.[3,4]). (iv) If $\chi^{(2)}$ is zero there is no coupling to $\phi$ in Eq. (4a). In this case the standard multi-dimensional NLS equation is obtained as a special "limiting" case. (v) If the incident field is polarized along both principal axes of the material, then both transversal components of the fundamental and dc field play an important role, and novel systems of coupled (3+1)-dimensional NLSM equations can arise [10].

It is also worth discussing what results are known for the $(2+1)$-dimensional NLSM equations which arise in water waves [6]: (i) one-dimensional solitons are unstable to slow transverse perturbations; (ii) for various choices of signs in the equations the solutions can blow up in finite time; (iii) for certain choices of signs and magnitude of the coefficients the equations are known to be integrable; (iv) in the integrable case stable localized pulses which are driven by the appropriate mean fields are known to exist $[16,17]$.

Most of these issues are also relevant in our context. In fact, one the main purposes of this work is to show that stable localized multi-dimensional pulses exist even in the more general non-integrable case. 


\section{Non-dimensionalization and rescalings}

If the $Y$ dependence of $A$ and $\phi$ is neglected, or if it is already taken into account when dealing with the linear modes (e.g. in a waveguide configuration), Eqs. (4a) and (4b) become effectively ( $2+$ 1)-dimensional. Then, upon non-dimensionalization, rescalings and rotation to the characteristic reference frame of Eq. (4b), the optical NLSM equations in the case of anomalous dispersion $\left(k^{\prime \prime}<0\right)$ can be rewritten as

$$
\begin{aligned}
& i q_{\zeta}+\left(1-\theta_{1}\right)\left(q_{\xi \xi}+q_{\tau \tau}\right)+2 \theta_{1} q_{\xi \tau}+\left(\theta_{2}|q|^{2}+Q\right) q=0, \\
& Q_{\xi \tau}=\left(|q|^{2}\right)_{\xi \xi}+\left(|q|^{2}\right)_{\tau \tau},
\end{aligned}
$$

where $q=A / A_{0}, Q=\phi / \phi_{0}, \xi=\left(X / X_{0}-T / T_{0}\right) / \sqrt{2}, \tau=\left(X / X_{0}+T / T_{0}\right) / \sqrt{2}, \zeta=Z / Z_{0}$ and $q_{0}, \phi_{0}$, $X_{0}, Z_{0}$ and $T_{0}$ are appropriate normalization units. In Eqs. (5a) and (5b) all the properties of the material are absorbed into the values of the constants $\theta_{1}$ and $\theta_{2}$, which are given by $\theta_{1}=\left[1-\left(r_{x, 1} / r_{x, 0}\right)\left(\left|s_{x}\right| / k_{x}\left|k_{x}^{\prime \prime}\right|\right)\right] / 2$ and $\theta_{2}=\left[\left(M_{x, 1} / M_{x, 0}-2 N_{+}\right] / N_{-}\right.$, with $N_{ \pm}=\left(N_{x, 1} /\left|s_{x}\right|\right) \pm\left(N_{x, 2} / r_{x, 0}\right)$. The integrable case corresponds to $\theta_{1}=\theta_{2}=0$. Eq. (5b) can be readily solved by expressing the dc field as $Q=U+V$, with

$$
U(\xi, \tau, \zeta)=\int_{\tau_{*}}^{\tau}\left(|q|^{2}\right)_{\xi} \mathrm{d} \tau+u_{*}(\xi, \zeta), \quad V(\xi, \tau, \zeta)=\int_{\xi_{*}}^{\xi}\left(|q|^{2}\right)_{\tau} \mathrm{d} \xi+v_{*}(\tau, \zeta),
$$

and where $u_{*}(\xi, \zeta), v_{*}(\tau, \zeta)$ are two arbitrary integration constants. If $\left(\xi_{*}, \tau_{*}\right)=(-\infty,-\infty)$, the corresponding two functions, which we call $u_{\infty}$ and $v_{\infty}$, assume the role of boundary conditions of the dc field $Q=U+V$. An alternative but equivalent choice which we will use in the following is $\left(\xi_{*}, \tau_{*}\right)=(0,0)$, corresponding to functions $u_{0}$ and $v_{0}$. It is clear that there is a one-to-one correspondence between any choice for $u_{\infty}$ and $v_{\infty}$ and any choice for $u_{0}$ and $v_{0}$. As demonstrated below, the functions $u_{*}, v_{*}$ play a key role in the dynamics of the pulse.

\section{Special solutions: integrable case}

In the integrable case (that is, when $\theta_{1}=\theta_{2}=0$ ), stable localized multi-dimensional pulses (sometimes called dromions) are known to exist, which are driven by the mean field through a proper choice of boundary conditions. Explicitly, the one-dromion solution of Eqs. (5a) and (5b) with $\theta_{1}=\theta_{2}=0$ is given by $[16,17]$

$$
q\left(x_{1}, x_{2}, t\right)=G\left(x_{1}, x_{2}, t\right) / F\left(x_{1}, x_{2}, t\right),
$$

where, for convenience, we set $x_{1}=\xi, x_{2}=\tau$ and $t=\zeta$, and where $G\left(x_{1}, x_{2}, t\right)=\rho \mathrm{e}^{\eta_{1}+\eta_{2}+i\left(\phi_{1}+\phi_{2}\right)}$, $F\left(x_{1}, x_{2}, t\right)=1+\mathrm{e}^{2 \eta_{1}}+\mathrm{e}^{2 \eta_{2}}+\gamma \mathrm{e}^{2\left(\eta_{1}+\eta_{2}\right)}$, with $\eta_{j}\left(x_{j}, t\right)=k_{j}\left(x_{j}-x_{0, j}-2 \omega_{j} t\right), \phi_{j}\left(x_{j}, t\right)=\omega_{j}\left(x_{j}-\right.$ $\left.x_{0, j}\right)-\left(\omega_{j}^{2}-k_{j}^{2}\right) t, j=1,2$, with $\rho=2 \sqrt{2(\gamma-1) k_{1} k_{2}}$, and with $k_{j}, \omega_{j}, x_{0, j}$ arbitrary real parameters. The constant $\gamma$ determines the overall amplitude, the parameters $k_{j}$ the width of the pulse in each respective direction, and the $\omega_{j}$ the Cartesian components of the velocity, while the $x_{0, j}$ determine the dromion position. The "potentials" $U\left(x_{1}, x_{2}, t\right)$ and $V\left(x_{1}, x_{2}, t\right)$ are obtained by integrating Eqs. (6) subject to the boundary conditions

$$
u_{\infty}\left(x_{1}, t\right)=2 k_{1}^{2} \operatorname{sech}^{2} \eta_{1}, \quad v_{\infty}\left(x_{2}, t\right)=2 k_{2}^{2} \operatorname{sech}^{2} \eta_{2} .
$$


The resulting expressions are

$$
U\left(x_{1}, x_{2}, t\right)=2\left(\ln F\left(x_{1}, x_{2}, t\right)\right)_{x_{1} x_{1}}, \quad V\left(x_{1}, x_{2}, t\right)=2\left(\ln F\left(x_{1}, x_{2}, t\right)\right)_{x_{2} x_{2}},
$$

Fig. 1a shows a typical (stationary) dromion solution, corresponding to $k_{1}=k_{2}=1, \omega_{1}=\omega_{2}=0$ and $\gamma=9$. The corresponding dc field $Q=U+V$ is shown in Fig. $1 \mathrm{~b}$ (note the non-zero boundary conditions $u_{\infty}\left(x_{1}\right)$ and $v_{\infty}\left(x_{2}\right)$ corresponding to Eq. (8)). The pulse is located at the intersection of $U$ and $V$.

\section{Special solutions: general case}

When $\theta_{1}$ and/or $\theta_{2}$ are not zero, there are no localized analytical solutions available, and one must resort to numerical simulations. We integrated Eq. (5a) with a two-dimensional second-order split-step method and Eq. (6) with a second-order numerical quadrature routine, for a number of different values of $\theta_{1}$ and $\theta_{2}$. Fig. 1a shows a typical stationary pulse in the integrable case $\theta_{1}=\theta_{2}=0$, while Fig. $1 \mathrm{~b}$ represents the corresponding dc field $Q=U+V$ obtained from Eq. (6) with $u_{\infty}, v_{\infty}$ given by Eq. (8). If the pulse
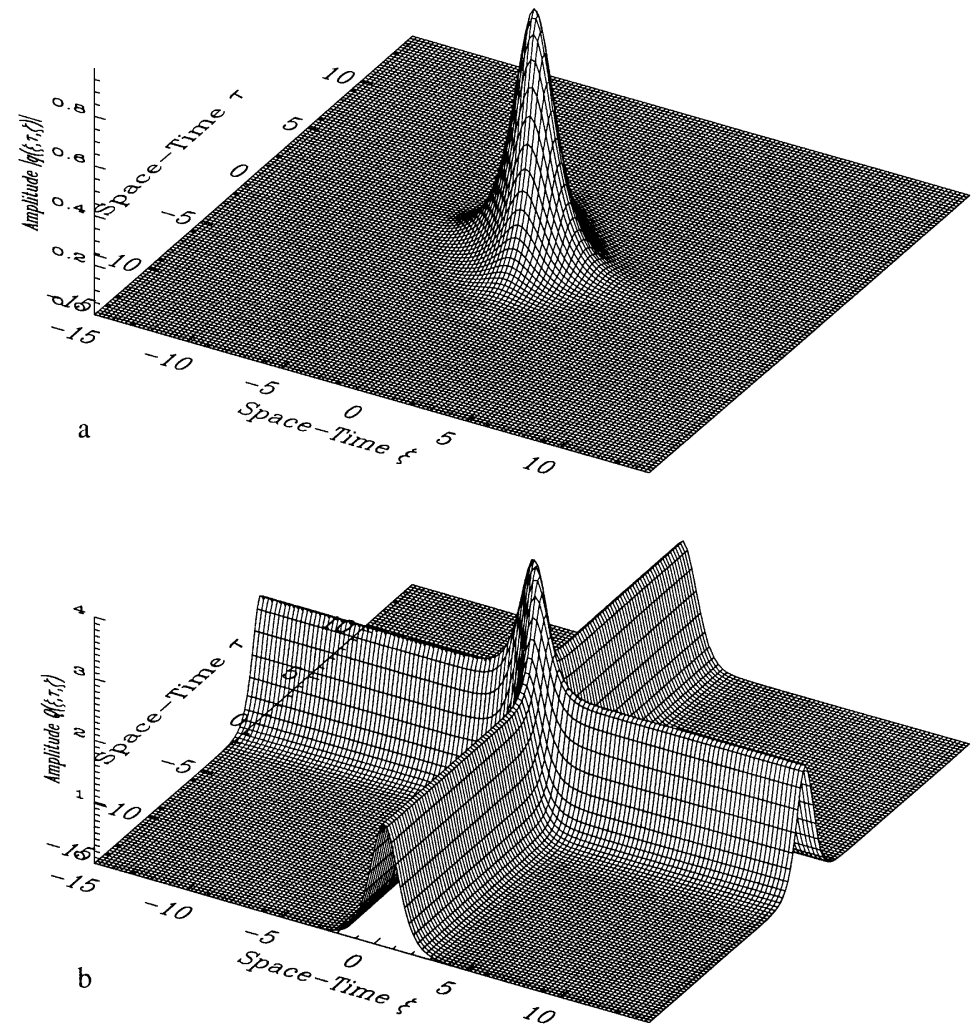

Fig. 1. The stationary dromion solution of the integrable case $\left(\theta_{1}=\theta_{2}=0\right)$ : (a) the optical pulse $|q(\xi, \tau, \zeta)|$; (b) the dc field $Q(\xi, \tau)=U(\xi, \tau)+V(\xi, \tau)$ associated with $q$. 


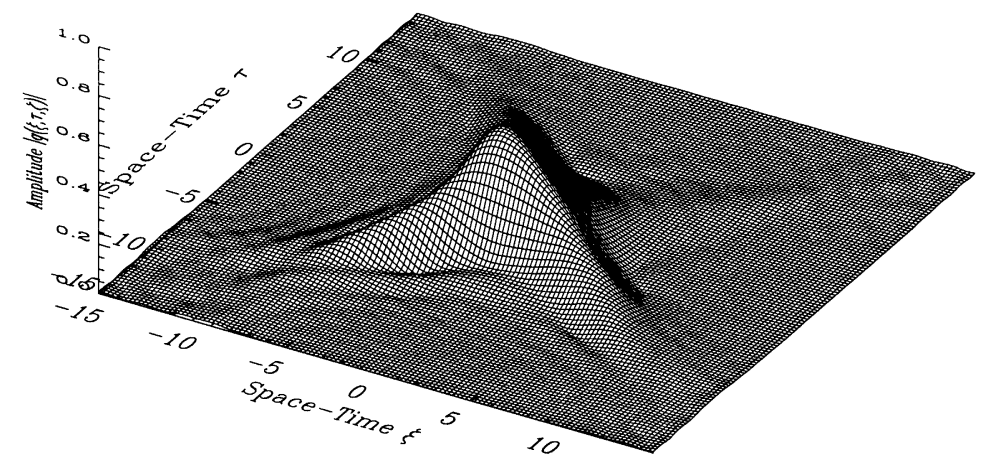

Fig. 2. The output pulse at $\zeta_{\text {out }}=2$, for $\theta_{1}=\theta_{2}=0.4$, with same initial condition as in Fig. 1a but with zero boundary conditions.

shown in Fig. 1a is used as initial condition for $\theta_{1}, \theta_{2} \neq 0$, and the same boundary conditions as in Fig. 1b are used for the dc fields numerical simulations show that, even though some radiation is produced, a localized pulse similar to the one in the integrable case persists for a long propagation distance. On the other hand, Fig. 2 shows the output produced after just two propagation distances by the same input pulse as in Fig. 1a if the boundary conditions $u, v$ for the dc fields are zero (i.e. $u_{\infty}=v_{\infty}=0$ ), for $\theta_{1}=\theta_{2}=0.4$. In this case the pulse decays very quickly, and no localized asymptotic state is obtained (note that the pulse disperses along directions which are the analog of the Mach lines associated with the propagation of a supersonic disturbance in a classical fluid). It is, therefore, clear that, even in the more general situation $\theta_{1} \neq 0, \theta_{2} \neq 0$, the dc fields can stabilize the optical pulses, which otherwise would disperse away very quickly without the presence of non-zero boundary conditions. Similar results were found for a wide range of values of $\theta_{1}$ and $\theta_{2}$.

A further improvement can be achieved if $u_{0}, v_{0}$ are used instead of $u_{\infty}, v_{\infty}$. In this case, generalized stationary solutions are found to exist even when $\theta_{1}$ and $\theta_{2}$ are significantly different from zero. To find these solutions, we integrated Eqs. (5a) and (6) with $\theta_{1}, \theta_{2} \neq 0$, using the dromion solution of the integrable case as initial condition and inserting absorbing boundaries at the edges of the two-dimensional grid to remove the radiation shed by the pulse. After the pulse has reached an asymptotic state, we removed the absorbing boundaries and let the pulse evolve according to the NLSM equations, to verify that we have obtained a stationary solution. As an example, in Fig. 3a we show the stationary pulse corresponding to $\theta_{1}=\theta_{2}=0.4$, while Fig. $3 \mathrm{~b}$ is relative to the case $\theta_{1}=0, \theta_{2}=-1$ (in particular, this last case implies that, even for defocusing self-interaction, the presence of non-zero asymptotic mean fields is sufficient to maintain a localized state). These findings suggest that stable localized multi-dimensional pulses are not unique to integrable systems; rather, they are a generic feature of forced evolution equations of this type.

When $\theta_{1}$ and $\theta_{2}$ are non-zero, the stationary pulses differ significantly from the corresponding solution of the integrable case. In particular, a numerical study of the equations reveals that $\theta_{1}$ affects the shape of the pulse, while $\theta_{2}$ controls its amplitude. More precisely, we find that, starting from a fixed initial condition, for increasingly negative values of $\theta_{2}$ (implying strong defocusing), the amplitude of the asymptotic stationary state decreases, while for increasing positive values of $\theta_{2}$ (implying strong focusing) the final amplitude of the solution increases until, for large enough values of $\theta_{2}$, the pulse does not asymptote to a stationary state anymore, and, presumably, higher order solutions are obtained. 

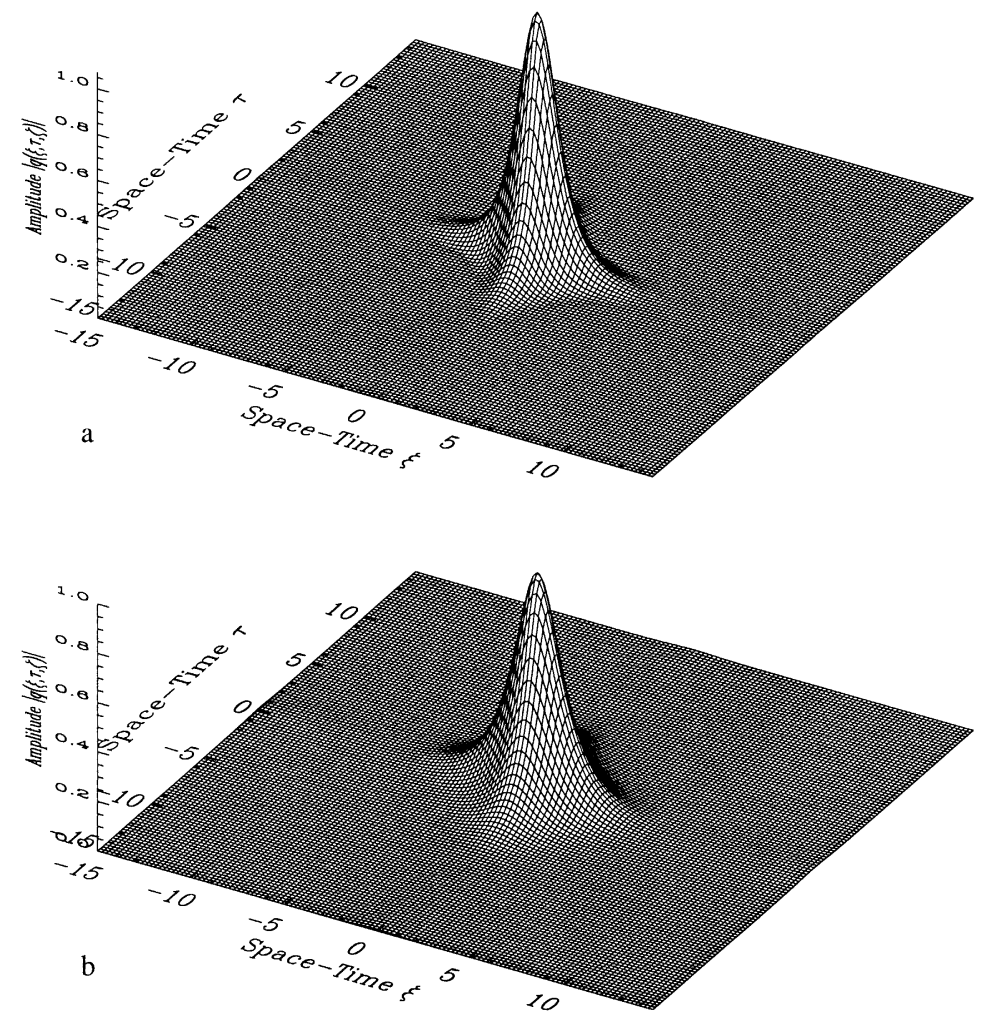

Fig. 3. Stationary solutions of the optical NLSM equations in the non-integrable case: (a) $\theta_{1}=\theta_{2}=0.4$; (b) $\theta_{1}=0, \theta_{2}=-1$.

Some of these features can be explained on the basis of simpler one-dimensional models. To this aim, we consider the parametrically forced NLS equation

$$
i q_{t}+\frac{1}{2} q_{x x}+\left(V(x)+\theta|q|^{2}\right) q=0 .
$$

As initial condition and forcing potential we take respectively $q_{0}(x)=a \operatorname{sech} x$ and $V(x)=A \operatorname{sech}^{2} x$. We solve Eq. (10) numerically for a number of values of $A, a$ and $\theta$. By comparison with the usual NLS equation it is clear that, if $A+\theta a^{2}=1$ (that is, if $a=\sqrt{(1-A) / \theta}$ ), the initial condition corresponds to the profile of the stationary state $q(x, t)=a \operatorname{sech} x \mathrm{e}^{i t / 2}$. This implies that, in the presence of a strong enough forcing potential ( $A>1$ in this case), stationary solutions also exist for $\theta<0$, however, the amplitude of these states decreases with $\theta$. For $\theta>0$ the picture is more complicated. In this case, looking for stationary solutions of the form $q(x, t)=f(x) \mathrm{e}^{i \lambda^{2} t}$, with $f(x)$ real, Eq. (10) leads to the following non-linear eigenvalue problem:

$$
f_{x x}+2\left(V(x)+\theta f^{2}-\lambda\right) f=0 .
$$

The number $N$ of discrete eigenvalues $\lambda_{j}$ in Eq. (11) depends of course on the potential $V(x)$, but also on the self-phase modulation coefficient $\theta$. If $N>1$, the solution of Eq. (10) may be expected to be a superposition of all the corresponding stationary modes. Since each of these modes has its own frequency, 
the overall pulse can be expected to undergo periodic or quasi-periodic oscillations, which corresponds to what is observed numerically in the two-dimensional system.

\section{Conclusions}

The dynamics of optical pulses in multi-dimensional media is characterized by new phenomena. In this letter we have studied the propagation of a single quasi-monochromatic optical pulse in media with quadratic non-linearity. In such media, mean fields play an important role in the dynamics. If the wave interactions are not resonant, the evolution of the pulse is often governed by NLSM-type equations. These equations are the $(3+1)$-dimensional analog (and generalization) in non-linear optics of the $(2+1)$-dimensional Benney-Roskes equations in water waves. In some circumstances the dynamics of the optical pulse depends critically on the boundary conditions chosen for the dc field. For appropriate choices of boundary conditions, stable localized multi-dimensional pulses can arise even in the non-integrable case. These findings suggest that stable localized multi-dimensional pulses are not unique to integrable systems; rather, they are a generic feature of non-linear evolution equations with forcing terms like those present in Eq. (6). In our case, the presence of small applied dc fields $\left(\mathrm{O}\left(\varepsilon^{2}\right)\right.$ in the perturbation expansion) can drive much larger optical pulses $(\mathrm{O}(\varepsilon)$ in the expansion). Preliminary studies indicate that the above described dynamical configuration could be realized experimentally, given the wide range of values of $\theta_{1}$ and $\theta_{2}$ over which stationary propagation occurs. It is expected that, since these values can be adjusted through linear material properties, proper design of the device structure will ensure pulse propagation within the desired regime. This possibility is particularly interesting because such experiments would allow the production of stable localized multi-dimensional optical pulses whose dynamics can be electrically controlled by modification of the relevant dc fields. These issues are currently under careful investigation and we will report on them in the near future.

\section{Acknowledgements}

We acknowledge P.A. Clarkson for useful discussions. This effort was partially sponsored by the Air Force Office of Scientific Research under grant number F49620-97-1-0017 and by the National Science Foundation, under grant number ECS-9800152.

\section{References}

[1] C. Bosshard, R. Spreiter, M. Zgonik, P. Günther, Phys. Rev. Lett. 74 (1995) 2816.

[2] A.A. Zozulya, D.Z. Anderson, Phys. Rev. A 51 (1995) 1520.

[3] A.G. Kalocsai, J.W. Haus, Opt. Commun. 97 (1993) 239.

[4] A.G. Kalocsai, J.W. Haus, Phys. Rev. A 49 (1994) 574.

[5] V.E. Zakharov, A.M. Rubenchik, Sov. Phys. JETP 38 (1974) 494.

[6] M.J. Ablowitz, H. Segur, Solitons and the Inverse Scattering Transform, SIAM, 1981.

[7] D.J. Benney, G.J. Roskes, Stud. Appl. Math. 48 (1969) 377.

[8] V.E. Zakharov, Sov. Phys. JETP 35 (1972) 908.

[9] M.J. Ablowitz, H. Segur, J. Fluid Mech. 92 (1979) 691.

[10] M.J. Ablowitz, G. Biondini, S. Blair, Phys. Lett. A 236 (1997) 520. 
[11] D.J. Benney, A.C. Newell, J. Math. Phys: (Stud. Appl. Math.) 46 (1967) 133. Stud. Appl. Math was called J. Math. Phys in 1967.

[12] A.V. Buryak, Y.S. Kivshar, S. Trillo, Phys. Rev. Lett. 77 (1996) 5210.

[13] C.B. Clausen, O. Bang, Y.S. Kivshar, Phys. Rev. Lett. 78 (1997) 4749.

[14] A.G. Kalocsai, J.W. Haus, Phys. Rev. E 52 (1995) 3166.

[15] A.G. Kalocsai, J.W. Haus, J. Opt. Soc. Am. B 14 (1997) 1680.

[16] M. Boiti, J.J. Leon, L. Martina, F. Pempinelli, Phys. Lett. A 132 (1988) 432.

[17] A. Fokas, P. Santini, Phys. Rev. D 44 (1990) 99. 